\title{
From Neurons to Brain: Adaptive Self-Wiring of Neurons
}

\author{
Ronen Segev and Eshel Ben-Jacob \\ School of physics and astronomy, \\ Raymond \& Beverly Sackler Faculty of exact Sciences, \\ Tel-Aviv University, Tel-Aviv 69978, Israel
}

April 20, 2021

\begin{abstract}
During embryonic morpho-genesis, a collection of individual neurons turns into a functioning network with unique capabilities. Only recently has this most staggering example of emergent process in the natural world, began to be studied. Here we propose a navigational strategy for neurites growth cones, based on sophisticated chemical signaling. We further propose that the embryonic environment (the neurons and the glia cells) acts as an excitable media in which concentric and spiral chemical waves are formed. Together with the navigation strategy, the chemical waves provide a mechanism for communication, regulation, and control required for the adaptive self-wiring of neurons.
\end{abstract}

\section{Introduction}

The brain is probably one of the most challenging and alluring complex system scientists can study [1]. And indeed, much effort has been devoted to brain studies from the physiological level of ionic channels to the philosophical level where questions about intelligence, self-awareness, and conciseness are discussed. Yet, one of the fascinating aspects about the brain has almost been completely ignored until recently. We refer to the process in which a collection of individual neurons are transformed into a functioning network with unique capabilitiesthe brain. This emergence process can not be totally determined by the stored genetic information. In a human brain, for instance, there are approximately 
$10^{11}$ neurons that form a network with more that $10^{15}$ synaptic connections. The precise structure of such a network can not be stored genetically. The human $D N A$ is composed of about $10^{9}$ bases, so it lacks sufficient memory for the detailed structure of a brain. The alternative extreme explanation, of total randomness, could not be correct as well. After all, we know that while on the micro level (up to about $1 \mathrm{~mm}$ ) the structure appears to be random, on the macro level (above $1 \mathrm{~cm})$ the brain's structure is quite deterministic. In addition, the brain structure varies from species to species, and, within a given species, some brain skills are inheritable (vs. learned). So clearly some elements of the brain structure must be dictated by stored genetic information.

At present we do not know exactly which information of the brain structure is stored, and the general question about the role of randomness vs. determinism during the emergent process is still open.

We describe here elements of a novel strategy for the emergent process involving interplay between randomness and determinism (via stored genetic information). Our proposed model is for simplified $2 D$ systems. As such, it is far from being a full description of the brain adaptive self-wiring. Yet, if tested and shown to be correct, it will provide an important step towards understanding the emergence process in a real brain.

A major obstacle in unraveling the fundamental principles of adaptive selfwiring is the complexity of the real brain. Hence much effort is devoted to in-vitro experimental studies of much simpler $2 D$ systems [2, 3]. In these experiments, neurons (primary or cell line) are placed on a Poly-L-Lysine (PLL) surface forming a simple $2 D$ system. The latter facilitates microscopic monitoring of the selfwiring and allows performing of parallel measurements of the electrical activity. The theoretical studies presented here are for such simpler $2 D$ systems.

Our model is composed of two main elements: 1. A navigation strategy for the micro level, i.e. length scale below $1 \mathrm{~mm}$ or about $10^{3}-10^{4}$ cells area in 
$2 D$ systems. 2. A mechanism for adaptive self-organization on the meso (about $1 \mathrm{~mm}-1 \mathrm{~cm})$ and macro scale (above $1 \mathrm{~cm})$.

The contemporary view is that the brain structure is essentially deterministic on a large scale but probabilistic on a small scale [1]. As a consequence the network has no optimal structure. But we believe that neural networks can be constructed in an optimal way, and this optimal way is derived from the biological mechanisms that construct the network. In any approach to the construction of the network there must be a precise strategy by which the neurons find each other to establish synaptic connections. At the beginning of the growth process the neurite has to migrate from it's own cell soma. The neurite migrates to the area in which it is supposed to form a synaptic connection. In this area there are many neurons, each of which is a possible target cell for the neurite. When the neurite approaches one of the possible target cells, with which it will finally form a synaptic connection, it has to be attracted to that cell soma.

The navigation strategy we propose is based on sophisticated means of chemical signaling for communication and regulations, including repulsive and attractive chemotaxis (chemotaxis is a movement based by a gradient of a chemical agent.) The strategy is described in section 2 and 3.

For reasons presented in section 5 we propose that the additional mechanism for self-wiring on the meso and macro scales is based on chemical waves. This proposal is inspired by the $c A M P$ waves employed during aggregation of Dictyostelium discoideum amoeba [4, 5] and is also motivated by the fact that chemical waves (including of $c A M P$ ) play role during the brain activity.

\section{Neurite as Amoeba with a tail}

Neurites are equipped for navigation with a unit called growth cone [6]. It is known that the growth cone is capable of measuring concentration and concentration gradients of chemicals [3, 7], including the gradients of repulsive and 
attractive chemotactic agents.

The movement of the growth cones appears to be a non-uniform random walk with the highest probability to move forward ("inertia") and a high probability to move backward ("retraction") [8, [] . The typical growth rate is of the order of micron/minute [10]. In the presence of a chemotactic agent, the movement is biased towards (attractive chemotactic) or away (repulsive chemotactic) from the chemical gradient. The movement is reminiscent of that of amoebae Dictyostelium discoideum 国 in the presence of chemotactic materials. For that reason R. Lumins refers to the growth cone and its neurite as "amoeba with a tail" 11.

In modeling the neurite navigation we were inspired by the bions model used in the study of amoebae aggregation [5] and by the communicating walker model used in the study of bacterial colonies [12, 13, 14]. In the model the growth cones are represented by walkers which perform off-lattice non-uniform random (biased) walk [15]. The chemical dynamics (e.g chemotactic agents, triggering field) are described by continuous reaction-diffusion equations solved on a tridiagonal lattice with a lattice constant $a_{0}=10 \mu \mathrm{m}$. Each of the soma is represented by a stationary (not moving) unit occupying one lattice cell. The neurite are simply defined as the trajectory performed by the growth cone.

\section{Chemotactic Navigation}

We assume that each of the soma cells continuously emits a repulsive agent whose concentration is denoted by $R$. In the model, $R$ satisfies the following reaction diffusion equation:

$$
\frac{\partial R}{\partial t}=D_{R} \nabla^{2} R+\Gamma_{R} \sum_{\text {soma }} \delta\left(\vec{r}-\overrightarrow{r_{j}}\right)-\lambda_{R} R
$$

Where $D_{R}$ is the diffusion coefficient, $\lambda_{R}$ is the spontaneous decomposition rate and $\Gamma_{R}$ is the emission rate by the soma cells.

When a neurite first sprouts it is mainly affected by the repulsive agent and moves away from its "mother" soma cell. It then continues to move on a trajectory 
which maximizes the distances from the surroundings soma cells (Fig 1a).

When a neurite reaches a specific length determined by its soma cell in a manners described below, it does two things: 1. It switches its chemotactic sensitivity from sensitivity to the repulsive agent to sensitivity to the attractive. 2. It emits a quantum of a triggering material (which satisfies an equation similar to eq. 1. Soma cells in the neighborhood respond by emitting a quantum of attractive agent if they sense an above threshold concentration of the triggering material. As a result, the growth cone moves towards the soma cell with the strongest attractive response (typically, the one closest to the growth cone, see Fig $1 \mathrm{~b})$. The above features are included in the model, as we show in detail in Ref [15].

\section{Simulation of the chemotactic navigation}

As was mentioned, the reaction-diffusion equations are solved on a tridiagonal lattice with a lattice constant $a_{0}=10 \mu m$. Thus, the fact that the soma occupies one lattice cell is in agreement with their typical size. The typical size of the simulated system is about $200-400 a_{0}$ and the distance between cells is about $25 a_{0}$. A typical diffusion coefficient $D$ of the chemicals is of the order of $10^{-6}-$ $10^{-7} \mathrm{~cm}^{2} / \mathrm{sec}$ [16. Time is measured in units of $10 \mathrm{sec}$, thus the dimensionless diffusion coefficients are of the order of 10 .

In the simulations, the walkers growth rate is about $1 / 64$ in dimensionless units which is in agreement with the measured growth rate. To demonstrate the efficiency of our proposed chemotactic navigation strategy, we consider two soma cells with a barrier in between (Fig 2). The cell on the right is a "normal" soma cell, while the one on the left is a "variance" which is incapable of emitting neurites. The choice of only one cell emitting neurites is in order to make the wiring pattern more transparent. First, in the absence of chemotactic communication (Fig 2a) the barrier prevents the formation of synaptic connections between the 
two cells. When included, the chemotactic communication enables to over come the barrier effect and the two cells are wired as is shown in Fig $2 \mathrm{~b}$.

We have mentioned that the growth cones" "sensitivity switch" (from repulsive to attractive agent) is controlled by the soma. Actually, as is discussed in details in Ref [15], we propose that the "sensitivity switch" is directly controlled by the metabolic state of the growth cone which is indirectly controlled by the soma. In the model, the metabolic state is represented by an "Internal energy", which is supplied at a given "feeding" rate by the soma. The rate of consumption of the internal energy is proportional to the neurites' length. Thus, for a given rate of feeding the "internal energy" will decrease for a given length. At this length the growth cones switches its chemotactic sensitivity. It means that the soma controls the "sensitivity switch" of the growth cone via adjustment of its feeding rate. This way the soma can control the distance at which its various neurites form the synaptic connections. An example is shown in fig 3. Here the central cell is "normal" and all the target cells are "variance" cells. The first two neurites are fed at a low rate. Hence they are connected to the nearest neighbors. The third neurite is fed at a higher rate. Thus it forms a connection further away.

\section{A need for additional mechanism}

So far we have described a navigation strategy for the microlevel. This strategy on its own is limited in the variety and complexity of the wiring patterns it can produce (see Fig 4). In these figures we see that the patterns have simple homogeneous structure of connectivity. Hence the genetic effect on the wiring patterns produced by this strategy has to be limited. At the same time it is known that the brain has a specific organization on the macro-scale (centers of activity over $1 \mathrm{~cm}$ ) which is genetically determined. In general it is not known what happens on the meso-scale between the apparent randomness of the microscale and the deterministic organization of the macro-scale [1]. We believe (unlike 
Ref [1]) that the micro-structure is not entirely random nor the macro-structure is

fully deterministic. But more important, we expect a gradual transition from the meso-structure to the macro-structure with partial deterministic organization on the meso-scale. If so, there should be an additional mechanism (to the navigation strategy) to enable meso and macro scales self organization.

\section{The excitable media mechanism}

It is well established that chemical waves, which affect the electrical activity of neurons, can propagate through out the cortex 117, 18, 19, 20, 21]. This brings to mind that such chemical waves can regulate the wiring process over the meso and macro scales.

A classical example of self-organization mediated via chemical waves is the aggregation of the slime mold Dictyostelium amoebae during starvation. These microbes collectively form an excitable media which produces spiral waves of $c A M P$. The movement of the individual organism is biased by the local gradient of the $c A M P$ (chemotaxis response). The result is regulated movement towards the center of the colony along well defined stream lines [4, 5].

The embryonic media is composed of two main cell types: neurons and glia. The Glia cells are the majority of the cell population in the brain ( $90 \%$ of the brains cells are glia) [6]. It is widely accepted that the glia give the structural support to the brain and they play part the main in the neurons nutrition. Inspired by the aggregation of slime mold amoebae we propose additional role to the glia: they acts as an excitable media and form chemical waves of at least one additional agent refered to as Glia Chemical Agent (GCA) needed for self-wiring on the meso and macro scale. The GCA (which can also be $c A M P$ ) plays a similar role to that of the $c A M P$ during amoebae aggregation. It acts as an additional chemotactic agent for the growth cone movement.

To model the glia role as excitable media we place randomly additional sta- 
tionary units (representing the glia) on the $2 \mathrm{D}$ hexagonal lattice with density $\rho_{G}$. Typically the $2 D$ density $\rho_{G}$ should be $0.05-0.2$ in numbers. Each Glia unit can be in one of three possible states: 1. rest state, 2. excitable state, and 3. refractory state.

When the unit is at rest state, at each time step, it measures the concentration of the chemotaxis at its location. Once the concentration is above a threshold level, the unit becomes excited (enter state 2) and starts to emit a quantum of GCA during $\tau_{1}$ time units. Then it enters the refractory state and it is immune to further excitation. After another $\tau_{2}$ time units the state progresses to rest state.

Numerical experiment of these system reveal [5] two basic patterns of waves that propagate in the medium. In figure $5 \mathrm{a}$ we show concentric waves that propagate in response to a periodic pacemaker at the center of the grid, and In figure $5 \mathrm{~b}$ we show a spiral waves that propagate in the media in response to appropriate initial conditions.

As we mentioned the length and time units of our simulation corresponds to $10 \mu m$ and $10 s$ respectively. To test the consistency of the model we compare the velocity of waves propagation in the simulation with the experimental one. The measured velocity of $c A M P$ waves is about $300 \mu \mathrm{m} / \mathrm{min}$ [4] and the measured velocity of the waves in the cortex is about $3000 \mu \mathrm{m} / \mathrm{min}$ [21.

The velocity of the waves in simulation is $10 a_{0}$ per 1 time step which corresponds to $10^{-2} \mathrm{~cm} / \mathrm{s}$. This is in agreement with the wave velocity measured in experiments $(\sim 0.06 \mathrm{~cm} / \mathrm{s})$.

We further assume that above a threshold level of the GCA concentration it acts as an additional chemotactic agent (either repulsive or attractive) on the movement of the walkers (growth cones). In Fig 6 we demonstrate the GCA effect in a system of 100 cells. The soma are restricted to emit only one neurite each in order to make the wiring patter more transparent to the eye. In Fig 6a there are no chemical waves and indeed the wiring pattern "looks" random . Next we 
placed a pacemaker at the center to produce concentric waves. In Fig $6 \mathrm{~b}$ we took the GCA to act as an attractive chemotaxis agent, and in Fig 5c to act as a repulsive one.

Finally in Fig 7 we demonstrate the effect of spiral wave. In this case the system is composed of 1250 cells, each soma emits two neurites and repulsive response is taken. In order to make the pattern of the network more transparent to the eye we line up neurites, i.e we draw a line between the cell soma of and the walkers position (fig 8).

\section{Conclusions}

We have presented a navigation strategy for micro-level network organization and an excitable media based wiring mechanism for the meso and macro levels organization. These mechanisms leads to the formation of neural networks with different structures, which can be genetically dependent. Our results raise two fundamental issues: 1. One needs to develop characterization methods (beyond number of connections per neurons) to distinguish the various possible networks. 2. The relations between the network organization and its computational properties and efficiency.

To clarify what we mean by characterization method, we consider the following example in which the network is mapped into a directed graph. Each neuron is represented by a vertex and each synaptic connection between neurons is represented by a directed edge between the two vertices representing the neurons. We can map the graph structure into an adjacency matrix where there is 1 in the $\mathrm{i}, \mathrm{j}$ entry of the matrix if there is a directed edge between the $\mathrm{i}$ and $\mathrm{j}$ vertices

and 0 otherwise. Then using an algebraic method, such as spectral theory of matrices, we can analyze the adjacency matrix of the graph which characterize the network. In other word, just as astronomers study spectra to determine the make-up of distance stars, we try to deduce the principle properties and structure 
of graph from its adjacency matrix spectrum [22].

The next step of the endeavor presented here is to include the effect of the network electrical activity on its self-wiring. Once some initial synaptic connection are formed, the network begins its electrical activity. It is natural to expect that from this point on the wiring dynamics depend on the electrical activity. An example to such a dependence is to assume that the neurons emit chemoattractant while it bursts a train of spikes. In addition we assume that a growth cone is more sensitive to the chemoattractant when its soma bursts. Using such navigation strategy we expect that neurons with correlated electrical activity will have higher probability to form a synaptic connection, as is shown in fig 9 .

To conclude, we are still far from understanding the emergence of a functioning brain from a collection of neurons. Yet we believe our studies provide a useful first step towards this goal.

\section{Acknowledgments}

The research was supported in part by grant No. BSF 92-00051 from the Israel USA binational foundation, by grant No. 593-95 from the Israeli Academy of Science, the Siegl Prize for Research, Sackler institute and The Adams Super Center.

\section{References}

[1] M. Abeles. Corticonics. Cambride University Press, 1991.

[2] B. Dwir, G. De Stasio, A. Sadeghi, D. Mercanti, M. Teresa Ciotti, G. Margaritondo, and E. Kapon. Directional growth of neuron networks on poly-llysine patterns. preprint, 1996.

[3] M. Tessier-Lavigne and C.S. Goodman. Science, 274:1123, 1996. 
[4] P.C. Newell. Cellular communication during aggregation of dictyostelium. J. of General; Microbiology, 104:1, 1978.

[5] H. Levine D. Kessler. Phys.Rev.E, 48:4801, 1993.

[6] I.B. Levitan and L.K. Kaczmarek. The Neuron. Oxford University Press, 1991.

[7] M. Tessier-Lavigne and M. Placzek. Target attraction: are developing axons guided by chemoatropism. TINS, 14:303, 1991.

[8] E. Braun. personal communication, 1997.

[9] H.M. Buettner, R.N Pittman, and J.K. Ivins. A model of neurite extension across regions of nonpremissive substrate: Simulations based on experimental measurement of growth cone motility and filopodia dynamics. Developmental Biology, 163:407, 1994.

[10] J.N. Barrett R.W. Gundersen. Neuronal chemotaxis: Chick dorsal-root axon turn toward high concentrations of nerve growth factor. Science, 206:1079, 1979.

[11] R. Lumins. personal communication, 1997.

[12] E. Ben Jacob et al. Complex bacterial patterns. Nature, 373:566, 1995.

[13] E. Ben-Jacob, O. Schochet, A. Tenenbaum, I. Cohen, A. Czirok, and T. Vicsek. Generic modeling of cooperative growth patterns in bacterial colonies. Nature, 368:46, 1994.

[14] E. Ben-Jacob. From snowflake formation to growth of bacterial colonies ii:cooperative formation of complex colonial patterns. Contemporary Physics, 38:205, 1997. 
[15] E. Ben-Jacob R. Segev. Self-wiring of neural networks. Phys. Lett. A, 237/45:307-313, 1998.

[16] G.J. Goodhill. A mathematical model of axon guidance by diffusible factors. Advances in Neural Information Processing Systems, 10, 1998.

[17] J.D. Murray. Mathematical Biology. SPRINGER-VERLAG, 1989.

[18] V.M Fernandes de Lima et al. Self-sustained spreading depression in the chiken retina and short term neuronal glial interaction within the gray matter neuropil. Brain Research, 614:45, 1993.

[19] S. Gooall K. Revett, E. Ruppin. Spreading depression in focal ischmia: A computational study. Beahvioral/System Neurosience, 1997.

[20] R.J. Do Carmo H. Martins-Ferrera. Retinal spreadimg depression and the extracellular milieu. Can.J.Physi.Pharma., 65:1092, 1987.

[21] J. Bures N.A. Grorelova. Spiral waves of spreading depression in the isolated chicken retina. J. of Neurobiology, 14:353, 1983.

[22] Fan R. K. Chung. Spectral Graph Theory. American Mathematical Society, 1997.

\section{Figure1}

\section{Figure2}

the effect of turning off the chemotactic communication. a. When we turn off the chemotaxis communication the walker are unable to reach their synaptic target. b. When we turn the communication on the walker can migrate around the barrier. 


\section{Figure3}

Simulations of a system composed of 30 cells. Only the cell at the center is "normal" and all other cells are "variance". The central cell has four nearest neighbor $(\mathrm{NN})$ cells and eight next nearest neighbor (NNN) cells. At the beginning of the growth the feeding rate is low. Thus the central cell is wired only to its NN cells. After The central cell forms two connections the "feeding" rate doubles. The new neurites navigate to the NNN cells. It demonstrates the manner in which the soma cell can regulates self-wiring.

\section{Figure4}

\section{Figure5}

Waves propagating in the media for $\rho_{B}=0.25, \tau_{1}=.8, \tau_{2}=5, D=10$, and $C_{T}=.001$. a. Spiral wave propagating in a $2 \mathrm{D}$ excitable media on a $200 \times 200$ grid. b. Target waves propagating in response to a pace make at the center of the grid (again $200 \times 200)$.

\section{Figure6}

In each network there are 100 cells which are restricted to emit only one neurite. a. There is no wave source at the center. The resulting network "looks" random. b. There is a pacemaker at the center of the grid. The GCA is assumed to be a repulsive agent. As a result the neurite migrate out from the center. c. Again a pacemaker at the center but now the GCA is assumed to be an attractive agent.

\section{Figure7}

The effect of spiral wave on the network structure. In the simulation we assumed the GCA is an attractive agent. The spiral wave propagates in response to appropriate initial conditions. 


\section{Figure8}

The same network as in figure 7 but now the final position of the growth cones is linked by a straight line in order to make the connections pattern more transparent.

\section{Figure9}

Example of the effect of the correlation in the electrical activity. The left and right neurons fire simultaneously and the first connection is formed between them. We remove the walkers which failed to create a synaptic connection in order to make the connections more transparent. 
This figure "figurela.gif" is available in "gif" format from: http://arxiv.org/ps/cond-mat/9806113v1 
This figure "figure1b.gif" is available in "gif" format from: http://arxiv.org/ps/cond-mat/9806113v1 
This figure "figure2a.gif" is available in "gif" format from: http://arxiv.org/ps/cond-mat/9806113v1 
This figure "figure2b.gif" is available in "gif" format from: http://arxiv.org/ps/cond-mat/9806113v1 
This figure "figure3.gif" is available in "gif" format from: http://arxiv.org/ps/cond-mat/9806113v1 
This figure "figure4.gif" is available in "gif" format from: http://arxiv.org/ps/cond-mat/9806113v1 
This figure "figure5a.gif" is available in "gif" format from: http://arxiv.org/ps/cond-mat/9806113v1 
This figure "figure5b.gif" is available in "gif" format from: http://arxiv.org/ps/cond-mat/9806113v1 
This figure "figure6a.gif" is available in "gif" format from: http://arxiv.org/ps/cond-mat/9806113v1 
This figure "figure6b.gif" is available in "gif" format from: http://arxiv.org/ps/cond-mat/9806113v1 
This figure "figure6c.gif" is available in "gif" format from: http://arxiv.org/ps/cond-mat/9806113v1 
This figure "figure7.gif" is available in "gif" format from: http://arxiv.org/ps/cond-mat/9806113v1 
This figure "figure8.gif" is available in "gif" format from: http://arxiv.org/ps/cond-mat/9806113v1 
This figure "figure9.gif" is available in "gif" format from: http://arxiv.org/ps/cond-mat/9806113v1 